\title{
Influence des systèmes de culture sur les risques d'érosion par ruissellement concentré. II. Evaluation des possibilités de maîtrise du phénomène dans les exploitations agricoles
}

\author{
François PAPY \& Jean BOIFFIN (*) \\ avec la collaboration de Claude DOLYER $\left({ }^{* *}\right)$ \\ I.N.R.A., Unité SAD, Cente de Recherches de Grignon-Mass\}-Paris, F 78850 Thiverval-Grignon \\ (*) I.N.R.A., Station d'Agronomie, rte Fernand-Christ, BP 101, F (02004 Laon Cedex \\ (**) I.N.R.A., Université de Roten, nue Thomas-Becket, F 76130 Mont-St-Aignan
}

RÉSUMÉ

\begin{abstract}
L"étude porte sur l'estimation des risques d"érosion par ruissellement concentré de différents systèmes de grande culture et les possibilités de les réduire par les techniques culturales. Les auteurs utilisent pour ce faire une procédure d'évaluation des risques adaptée au mode d'érosion par ruissellement concentré. Elle est fondée sur une modélisation empirique de l'ćvolution des états de surface du sol. Une discussion sur la compatibilité de plusieurs techniques anti-érosives avec la conduite des exploitations montre qu'on ne peut proposer les mêmes techniques à tous les types d'exploitations. La réduction du ruissellement par les techniques culturales ne peut être que partielle. aussi des aménagements spécifiquement conçus pour contrôler le ruissellement et réduire l'érosion sont-ils nécessaires
\end{abstract}

Mots clés additionnels: Bassin versant, fonctionnement des exploitations agricoles, Pays de Caux, techniques anti-írosives. possibilities within farms.

This paper presents an evaluation of the risk of erosion by concentrated runoff in different arable cropping systems and a discussion on the means of reducing this risk by agricultural practices. A convenient procedure has been developed to estimate the risk of concentrated flux erosion, consisting of an empirical model of change in the soil surface. The compatibility of certain soil conservation techniques with farm management was reviewed, and it did not appear possible to suggest the same proposals for all types of farming system. Runoff could be reduced by agricultural practices but to a limited extent, so specific management is necessary to control runoff and thus reduce erosion.

Additional key words : Catchment area, farming ststem, Pays de Caux, soil conservation technique.

\section{INTRODUCTION}

On observe des phénomènes d'érosion liés à la concentration du ruissellement dans certaines situations du nord-ouest de l'Europe (BoIfFIN et al., 1986; MonNier et al., 1986; AuzeT, 1987). Il sagit particulièrement de plateaux et collines limono-lœssiques à pentes douces, dont la topographie comporte un réseau de talwegs non drainés, et qui portent des systèmes de culture à faible proportion de prairie, pratiqués en grandes parcelles.

Sur des matériaux lœssiques, pour des valeurs de pentes supérieures à $4-5$ p. 100 , on observe fréquemment une érosion par formation de rigoles sur les versants (Savat \& de Ploey, 1982 ; Govers, 1985 ; De Ploey, 1986) ; mais en deçà de ce seuil, c'est dans les talwegs 
que se déclenche l'érosion par concentration du ruissellement émis par un vaste impluvium. Ce dernier processus, décrit par FosTer et al. (1985), SPOMER \& HJELMFELT (1986), domine dans les situations désignées cidessus où, par conséquent, les rigoles et ravines temporaires sont largement espacées les unes des autres. Il en résulte que la part de terre détachée par l'impact des gouttes de pluie, susceptible d'être récupérée et entraînée dans les rigoles, est faible. Aussi dans ce processus l'effet de protection du sol par le couvert végétal ne peut avoir les mêmes conséquences sur les pertes en terre que dans les situations d'érosion de versant à rigoles rapprochées (BOIFFIN et al., 1986). Les états de surface de l'impluvium sont déterminants dans la mesure où ils conditionnent le refus à l'infiltration, la détention d'eau en surface sous forme de flaques, la collecte du ruissellement et, par suite, la force de traction des écoulements dans le collecteur principal Ce dernier est le plus souvent le talweg, mais dans certains cas la fourrière peut jouer ce rôle.

Ayant étudié les conditions de déclenchement de l'érosion et les ayant reliées à la succession des états de surface induite par les systèmes de culture pratiqués (BOIFFIN et al., 1988), nous voulons utiliser ces résultats pour évaluer les possibilités de réduire la concentration du ruissellement et l'érosion. Mais d'emblée se pose la question suivante : dans quelle mesure doit-on modifier les systèmes de culture?

Dans les situations d'érosion par concentration du ruissellement citées plus haut, nous estimons que les inconvénients pour l'agriculteur (perte en terre, destruction partielle des cultures, gêne dans les travaux des champs) ne sont pas suffisants pour justifier des modifications de l'assolement. Ce dernier est principalement déterminé par les objectifs de production de l'agriculteur et ses moyens face à la conjoncture économicue. Aussi n'envisagerons-nous pas de faire des propositions qui remettraient en cause les grands choix de production.

Nous sommes alors conduits à poser deux questions :

1) Quelles sont les conséquences de ces choix sur le ruissellement et les risques d'érosion?

2) Est-il possible de proposer des techniques de limitation du ruissellement sans modifier pour autant. le choix des espèces cultivées et des surfaces attribuées à chacune d'elles?

On ne peut trouver dans la réalité un nombre suffisant d'exploitations agricoles à comparer en situations géomorphologiques identiques. Aussi les comparaisons devront-elles être établies non pas à partir de mesures ou d'observations directement faites sur le terrain, mais sur la base d'une simulation. Celle que nous proposons, en l'état actuel des connaissances, est pour partie qualitative.

\section{La méthode suivie comporte deux étapes :}

1) On commence par définir les systèmes de culture de la région d'étude et, pour pouvoir discuter de l'adoption par les agriculteurs de techniques anti-érosives, on distingue des types d'exploitation selon les objectifs des agriculteurs et les moyens de production (CAPILLON, 1986).

2) Dans un second temps, nous attribuons aux systèmes de culture définis, une note d'aptitude au ruissellement concentré. Elle résulte directement de l'analyse du rôle des états de surface dans le déclenchement de l'érosion (BoIfFin et al., 1988). Ce rôle est prépondérant car, dans la région d'étude, il existe une homogénéité suffisante des délcrimants de la stabilité structurale des sols, dont on sait a cflet sur la sensibilité à l'érosion (BOLLINE, 1977) et qu'on se situe dans des cas d'érosion chronique, sous faible intensité pluviométrique excluant les situations orageuses où l'effet de l'état initial du sol se trouve masqué.

Cette méthode servira à comparer des successions de cultures, différentes techniques et différents modes d'affectation des cultures aux parcelles d'un bassin versant sous l'hypothèse que les dimensions et formes des parcelles, la morphologie des bassins versants sont identiques. Sur chacun de ces thèmes seront discutées les possibilités d'adoption de pratiques diminuant le ruissellement et l'érosion.

L'ensemble des données utilisées est issu d'enquêtes réalisées dans le Pays de Caux (Seine-Maritime).

\section{MATÉRIEL ET MÉTHODES}

\section{A. La région d'étude}

Le Pays de Caux présente bien les caractéristiques géomorphologiques de situations à érosion par ruissellement concentré : vaste plateau à pentes douces (inférieures à 3 p. 100 sur les versants, de 2 à 6 p. 100 dans les talwegs) recouvert de limons battants (BoIFFIN et al., 1988). Le climat de type océanique y est très pluvieux. La moyenne pluviométrique annuelle est de $850 \mathrm{~mm}$ à Goderville, mais les pluies sont de faible intensité : en appliquant la formule de Montana, Pinguet (1985) calcule que dans cette région pour un pas de temps de 2 heures, le délai de retour d'une pluie de $20 \mathrm{~mm}$ est de 2 ans et celui d'une pluie de $29 \mathrm{~mm}$ de $10 \mathrm{ans}$.

Sur l'ensemble de la région, les surfaces toujours en herbe (STH) sont importantes, puisqu'au recensement général agricole (RGA) de 1979-1980 les terres labourables (TL) n'occupaient que 53 p. 100 de la surface agricole utile (SAU). Mais les localisations respectives de ces deux grandes modalités d'occupation du sol sont bien distinctes. Les prairies se cantonnent dans les fonds de vallée, sur les versants à forte pente et, sur le plateau, à l'entour des maisons et bâtiments d'exploitation, si bien que sur les parties amont des bassins versants, ce sont des systèmes de culture à espèces annuelles qui prédominent.

\section{B. Détermination des types d'exploitations et de systèmes de culture}

\section{Choir des exploitations étudiées}

Nous avons eu comme souci premier d'étudier des situations à risque. C'est pour cette raison que les trente exploitations enquêtées ont été choisies dans quatre cantons (Fontaine-Le Dun, St-Valéry-en-Caux, Criquetot, Montivilliers) parmi ceux qui ont le plus de terres 
labourables en position de plateau. Aussi dans notre échantillon le pourcentage consacré aux TL est-il bien supérieur à la moyenne régionale : le rapport TL/SAU varie de 55 p. 100 à 100 p. 100 avec une moyenne de 80 p. 100.

Mais au sein de l'échantillon, nous avons recherché la plus grande variété possible de systèmes de production, notre but étant d'inventorier des types de systèmes de culture différenciés. La taille des exploitations étudiées varie de 30 à 175 ha, avec une moyenne de 85 ha et un mode de 65 ha.

\section{Les types d'exploitation}

Les 30 enquêtes sur le fonctionnement des exploitations (CAPILLON \& MANICHON, 1988) nous ont permis de proposer une typologie. En ne retenant que les exploitations pour lesquelles le système de production semble fixé dans le moyen terme ( 3 à 5 ans), nous pouvons distinguer les types suivants (cf. tabl. 1):

a) Le type traditionnel est fondé sur l'exploitation d'un troupeau de vaches allaitantes et de sa suite, avec production de cultures de vente (betterave et lin). Dans la plupart des cas l'agriculteur âgé, proche de la retraite, ne disposant que de peu de main-d'œuvre ( 2 unités de travail humain (UTH)/100 ha) ne cherche pas à produire beaucoup par unité de surface. L'exploitant ayant besoin de grain pour l'alimentation du troupeau, l'escourgeon prend une place importante dans ces systèmes. L'assolement sur le plateau comprend généralement 50 p. 100 de céréales d'hiver et 50 p. 100 de cultures de printemps. On trouve ce type dans une grande gamme de taille de SAU.

b) Le type de grande culture avec peu de main-d'auvre (2 UTH/100 ha), comporte des cultures de vente mécanisables, peu exigeantes en travail, associées à un élevage se limitant à utiliser les prairies naturelles qu'on ne peut retourner. Sur le plateau il y avait, jusqu'à ces dernières années, 50 p. 100 de blé associé à du colza et des cultures de printemps (Succession II A). Mais en raison des rapports de prix, la tendance est à la diminution du blé au profit du pois (Succession II B).

c) Le type intensif à base de productions végétales se rencontre dans des exploitations de plus de 80 ha, dans lesquelles sont généralement associées deux générations d'exploitants. Aussi avec une main-d'œuvre abondante (4 UTH/100 ha) essentiellement familiale, l'objectif estil un fort revenu à l'hectare. L'élevage bovin n'existe plus; il est remplacé par un élevage hors-sol. Outre les cultures de vente courantes (betterave, lin, pois), ces systèmes produisent de la pomme de terre et, parfois, des légumes à forte marge brute. Sur le plateau, la surface en blé ne dépasse pas 30 p. 100 .

d) Le système intensif de production laitière avec cultures de vente se rencontre sur une grande gamme de taille d'exploitation. Toutes ont une forte maind'ouvre à valoriser (4 UTH/100 ha). Deux grands systèmes de culture y sont pratiqués : l'un constitué par les prairies naturelles et temporaires de longue durée autour des bâtiments d'exploitation, l'autre constitué par un système de culture dans lequel le maïs ensilage, la betterave fourragère et des cultures dérobées de raygrass d'Italie et de seigle occupent une place importante.

e) Le système intensif laitier sans cultures de vente en exemplaire unique dans notre échantillon, a cependant été retenu car il se distingue nettement des autres par l'introduction de prairies de courte durée dans la succession.

Les exploitations en cours d'évolution sont intermédiaires par rapport aux types précédents, mais les tendances générales sont à l'abandon de l'élevage et au retournement des prairies labourables, ainsi qu'à l'augmentation du pois et du colza au détriment du blé et de l'escourgeon.

\section{Procédure d'évaluation des risques de ruissellement concentré liés à un système de culture}

Du mémoire précédent (BoIfFin et al., 1988) nous pouvons déduire une procédure d'estimation de l'aptitude d'une parcelle à émettre, dans un état donné, du ruissellement et à le concentrer. Elle combine des critères déterminant l'infiltrabilité (stade de développement des croûtes sur les surfaces non affectées par les roues - BOIFFIN \& MONNIER, 1986 -, pourcentage de la superficie tassée en surface - FULLEN, 1985 ; RAUwS \& AUZET, 1988--), la détention superficielle de l'eau sous forme de flaques (rugosité) et la vitesse de concentration du ruissellement (présence d'un réseau de collecte orienté perpendiculairement au collecteur principal). Au tableau 2 des classes sont proposées pour les trois critères suivants :

- Développement des croûtes de surface, hors traces de roues $(\mathrm{A})$,

- Rugosité, hors traces de roues (B),

- Importance des traces de roues $(C)$,

ainsi que la correspondance avec des situations culturales typiques. La procédure de combinaison des critères précédents est donnée au tableau 3 . Le critère « trace de roues » y intervient par ses 2 fonctions sur l'infiltrabilité et la collecte du ruissellement et figure à la fois en ligne et colonne. Aussi certaines des cases du tableau, correspondant à des notes contradictoires entre lignes et colonnes, représentent des cas impossibles: elles sont vides. Les cases hachurées correspondent à des situations qui n'existent pas. Dans les autres sont portées des notes d'aptitude au ruissellement concentré permettant de classer des situations ayant même configuration morphologique, mêmes dimensions de parcelle, même orientation des traces de roues par rapport au talweg et ne se différenciant que par les états de surface (BOlFriN et al., 1988).

Appliqué à une parcelle, un système de culture induit une évolution des états de surface: instantanée sous l'action des façons culturales, plus progressive sous l'action des pluies.

Toute opération de travail du sol par fragmentation crée un état, qui, ne présentant pas de risque de ruissellement en raison des valeurs élevées d'infiltrabilité, peut être considéré comme un état initial 
TABLEAU 1

Les types de sistèmes de culture sur le platecut.

Types of cropping system on the platean.

\begin{tabular}{|c|c|c|c|c|c|c|c|c|c|c|c|c|c|}
\hline \multirow{2}{*}{$\begin{array}{l}\text { Dénomination } \\
\text { du type } \\
\text { d'exploitation }\end{array}$} & \multicolumn{11}{|c|}{ Assolement sur le plateau (1) } & \multirow{2}{*}{$\begin{array}{l}\text { Types de } \\
\text { successions } \\
\text { fréquentes }\end{array}$} & \multirow{2}{*}{$\begin{array}{l}\text { Numéro } \\
\text { de } \\
\text { réf. }\end{array}$} \\
\hline & & $\overbrace{\text { Esc }}^{\text {Eales }}$ & & $\overbrace{B F}^{\text {rave }}$ & L & $P$ & PT & Lég. & ME & $\begin{array}{l}\text { RGI } \\
18 \\
\text { mois }\end{array}$ & (DER) & & \\
\hline $\begin{array}{l}\text { Système } \\
\text { traditionnel }\end{array}$ & & 50 & & & $\leqslant 17$ & Faible & 0 & 0 & Faible & 0 & 0 & ${ }_{\mathrm{ME}}^{\mathrm{BS}}, \mathrm{BI} \cdots \mathrm{Esc}-\mathrm{L} \searrow_{\mathrm{P}}^{\mathrm{BS}}{ }^{\mathrm{Bl}}$ & $I$ \\
\hline $\begin{array}{l}\text { Système } \\
\text { de grande } \\
\text { culture } \\
\text { avec peu de } \\
\text { main-d'œuvre }\end{array}$ & $\begin{array}{l}30 \\
\grave{a} \\
50\end{array}$ & 0 & $\begin{array}{l}15 \\
\dot{a} \\
20\end{array}$ & 0 & $\begin{array}{l}15 \\
\text { à } \\
20\end{array}$ & $\begin{array}{l}15 \\
\mathbf{a} \\
30\end{array}$ & 0 & 0 & 0 & 0 & 0 & $\begin{array}{l}\mathrm{BS}-\mathrm{Bl}-\mathrm{P}-\mathrm{Bl}-\mathrm{L}-\mathrm{Bl} \\
\mathrm{BS}-\mathrm{P}-\mathrm{Bl}-\mathrm{L}-\mathrm{P}-\mathrm{Bl}\end{array}$ & II A \\
\hline $\begin{array}{l}\text { Système } \\
\text { intensif } \\
\text { a cultures } \\
\text { de vente }\end{array}$ & $\begin{array}{l}25 \\
\grave{a} \\
30\end{array}$ & 0 & $\begin{array}{c}25 \\
\dot{a} \\
30\end{array}$ & 0 & $\leqslant 17$ & Faible & $\begin{array}{l}15 \\
\text { à } \\
25\end{array}$ & $\begin{array}{l}0 \\
\dot{a} \\
10\end{array}$ & 0 & 0 & 0 & 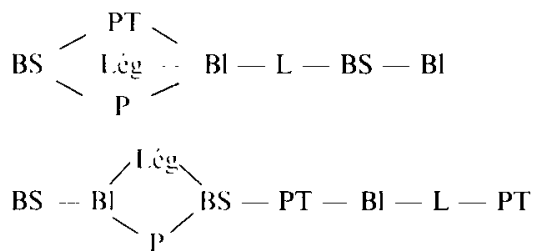 & $\begin{array}{l}\text { III } A \\
\text { III B }\end{array}$ \\
\hline $\begin{array}{l}\text { Système } \\
\text { intensif } \\
\text { laitier } \\
\text { avec cultures } \\
\text { de vente }\end{array}$ & $\begin{array}{c}25 \\
\dot{a} \\
30\end{array}$ & 0 & $\begin{array}{c}10 \\
\dot{a} \\
25\end{array}$ & 0 & $\leqslant 15$ & Event. & 0 & 0 & $\begin{array}{c}20 \\
\mathrm{a} \\
50\end{array}$ & () & Event. & $\begin{array}{l}\mathrm{ME}^{\mathrm{BS}} \mathrm{F}_{\mathrm{L}}^{\mathrm{ML}} \neq \mathrm{ME} \\
\mathrm{BS}-\mathrm{Bl}-\mathrm{ME}-\mathrm{P}-\mathrm{BI}-\mathrm{L}\end{array}$ & $\begin{array}{l}I V A \\
I V B\end{array}$ \\
\hline $\begin{array}{c}\text { Système } \\
\text { intensif } \\
\text { laitier } \\
\text { sans cultures } \\
\text { de vente }\end{array}$ & 20 & 20 & 0 & 0 & 0 & 0 & 0 & 0 & 40 & 20) & 0 & $\mathrm{ME}-\mathrm{Esc}-\mathrm{RGI}(18$ mois $)-\mathrm{ME}-\mathrm{Bl} \uparrow$ & $V$ \\
\hline
\end{tabular}

(1) Nous n'avons pas rapporté ici l'assolement sur les pentes ou en valléc. en grande partic consacré à l'herbe. Les pourcentages sont donnés par rapport aux Terres Labourables (TL).

Symboles:

Bl : Blé (wheat) : BF : Betterave fourragère (forage beet) : BS : Betterave sucrière (sugar bect); Esc : Escourgeon (winter barley) ; DER : Cultures dérobćes, en général Ray grass d'ltalic ou Seigle (indiquées par unc $\uparrow$ dans la succession de cultures) (Cover-crop, generally Italian ryegrass or rye) (shown by a $\uparrow$ in a cultural succession) : Lég: Légumes (vegetables) : L : Lin (flax): ME : Maìs ensilage (silage maize) ; PT : Pomme de terre (potato); P : Pois (pea); RGI : Ray Grass d'Italie (Italian rye grass)

(faciès F0). Selon la nature de l'opération, la rugosité est plus ou moins forte, le réseau des traces de roues plus ou moins développé. Sous l'action des pluies, des croûtes se forment en surface: d'abord structurales (faciès Fil), puis sédimentaires (faciès F2). L'infiltrabilité diminue jusqu'à des valeurs faibles ( $1 \mathrm{~mm} / \mathrm{h}$ environ), qui expliquent qu'à partir du faciès $F 2$ on observe du flaquage même pour de faibles intensités pluviométriques (BOIFFIN, 1984 ; Bolfilin \& MonNier, 1986). Parallèlement la rugosité, et par suite la détention de surface, diminue. Le risque de déclenchement du ruissellement augmente.

A chaque instant, on peut donc estimer l'aptitude d'une parcelle à générer du ruissellement concentré selon le tableau 3 si l'on sait classer les états de surface (tabl. 2). De la même façon, sur un ou plusieurs cycles culturaux, on peut estimer l'évolution de cette aptitude si l'on sait prévoir comment varient, au cours du temps, les critères d'état de surface. Or, à partir de références établies dans le Pays de Caux, nous sommes en mesure de relier l'apparition des croûtes sédimentaires (faciès F2) à des ordres de grandeur de cumul des pluies $(\Sigma \mathrm{Pmm})$ reçues depuis la date où la surface présente le faciès fragmentaire (FO). Nous avons admis que le seuil de déclenchement du faciès $F 2$ varie de 90 à $60 \mathrm{~mm}$ selon qu'une dessiccation du sol intervient ou non au cours de la formation des croûtes (BolfFin et al., 1986). A partir de dates conventionnelles de semis, qui correspondent à la fabrication par les outils du faciès fragmentaire $(\mathrm{F} 0)$, on peut prévoir approximativement les dates d'apparition de F2, comme indiqué au tableau 4. Par ailleurs le tableau 5 donne une loi de diminution de la classe de rugosité en fonction de $\Sigma$ Pmm à partir de références ćtablies par BoIfFIN (1984) sur un sol de même texturc.

Sur ces bases, et en adoptant le régime moyen des pluies de la région d'étude, nous pouvons proposer un schéma d'évolution des caractéristiques de la surface du sol et, par suite, de l'aptitude au ruissellement concentré. C'est ce que nous avons fait dans la figure 1 sur une succession betterave-blé prise comme exemple. 
L'aptitude à produire du ruissellement concentré est très élevée (note 3 ) juste après la récolte de la betterave (dégradation structurale poussée, note de rugosité faible, très nombreuses traces de roues); dans cette succession culturale, cette phase est de courte durée.

La note d'aptitude est nulle à très faible (note 0 ) dans deux cas :

1) les périodes post-semis, tant que la dégradation de surface n'a pas atteint le faciès des croûtes sédimentaires (F2),
2) la période d'interculture entre la récolte du blé et le semis de betterave. Au cours de cette dernière, bien que sous l'action des pluies la surface du sol puisse évoluer jusqu'au stade F2, la rugosité conférée au sol par le déchaumage, tout en s'atténuant au cours du temps, reste généralement suffisante pour empêcher la mise en mouvement de l'eau au-delà de distances métriques, tout au moins si l'on a pris soin, au cours du déchaumage, de ne pas créer des billons dans le sens de la pente.

Des risques intermédiaires (notés 2) existent en cours

TABLEAU 2

Les états de surface: codification et situations types.

Surface states: codification and typical situations

\begin{tabular}{|c|c|c|c|c|c|}
\hline \multirow{2}{*}{$\begin{array}{c}\text { Stade de } \\
\text { formation } \\
\text { des croûtes } \\
\text { (A) }\end{array}$} & Faciès & $\begin{array}{c}\text { FO } \\
\text { (Fragmentaire) }\end{array}$ & $\begin{array}{c}\mathrm{F} 3 \\
\text { (Croûte remaniée) }\end{array}$ & $\begin{array}{c}\text { Fl } \\
\text { (Croûte } \\
\text { structurale) }\end{array}$ & $\begin{array}{c}\text { F2 } \\
\text { (Croûte } \\
\text { sédimentaire) }\end{array}$ \\
\hline & Situations & Travail du sol & $\begin{array}{l}\text { Action physique } \\
\text { de dessiccation et } \\
\text { de gel } \\
\text { Action biologique }\end{array}$ & $\begin{array}{l}\text { Première phase de } \\
\text { dégradation par } \\
\text { les pluies }\end{array}$ & $\begin{array}{l}\text { Seconde phase de } \\
\text { dégradation par } \\
\text { les pluies }\end{array}$ \\
\hline \multirow[b]{2}{*}{$\begin{array}{l}\text { Rugosité } \\
\text { (B) }\end{array}$} & Classe & 1 & 2 & 3 & 4 \\
\hline & Situations & $\begin{array}{l}\text { Semis très battu } \\
\text { Etat très tassé } \\
\text { après récolte }\end{array}$ & $\begin{array}{l}\text { Semis assez battu } \\
\text { ou roulé } \\
\text { Semis très affiné }\end{array}$ & $\begin{array}{l}\text { Semis motteux peu } \\
\text { battu } \\
\text { Déchaumage } \\
\text { après récolte sans } \\
\text { résidus }\end{array}$ & $\begin{array}{l}\text { Labour } \\
\text { Déchaumage avec } \\
\text { résidus abondants }\end{array}$ \\
\hline \multirow[b]{2}{*}{$\begin{array}{l}\text { Traces de roue } \\
\text { en surface } \\
\text { (C) }\end{array}$} & Classe & 0 & 1 & 2 & 3 \\
\hline & $\begin{array}{l}\text { Situations } \\
\text { (Traces en \% de la } \\
\text { surface totale) }\end{array}$ & $\begin{array}{l}\text { Travail du sol } \\
(0 \%)\end{array}$ & $\begin{array}{l}\text { Semis de céréales, } \\
\text { pois, lin } \\
(5 \% \text { après traite- } \\
\text { ment })\end{array}$ & $\begin{array}{l}\text { Semis de betterave } \\
\text { et maïs } \\
\text { Plantation de PT } \\
(15 \text { a } 35 \%)\end{array}$ & $\begin{array}{l}\text { Récolte de BS et } \\
\text { PT }(70 \text { a } 80 \%)\end{array}$ \\
\hline
\end{tabular}

Dans les figures 1 et 2, ainsi que dans le tableau 3, les 3 critères d'état de surface sont désignés par les symboles $\mathrm{A}, \mathrm{B}$ ct $\mathrm{C}$.

TABLEAU 3

Procédure de classement de l'aptitude croissante au ruissellement concentré (répartie en 4 classes, notées 0, I, 2, 3).

Procedure for grading the risk of concentrated runoff (on a 4-point scale-0, 1, 2, 3).

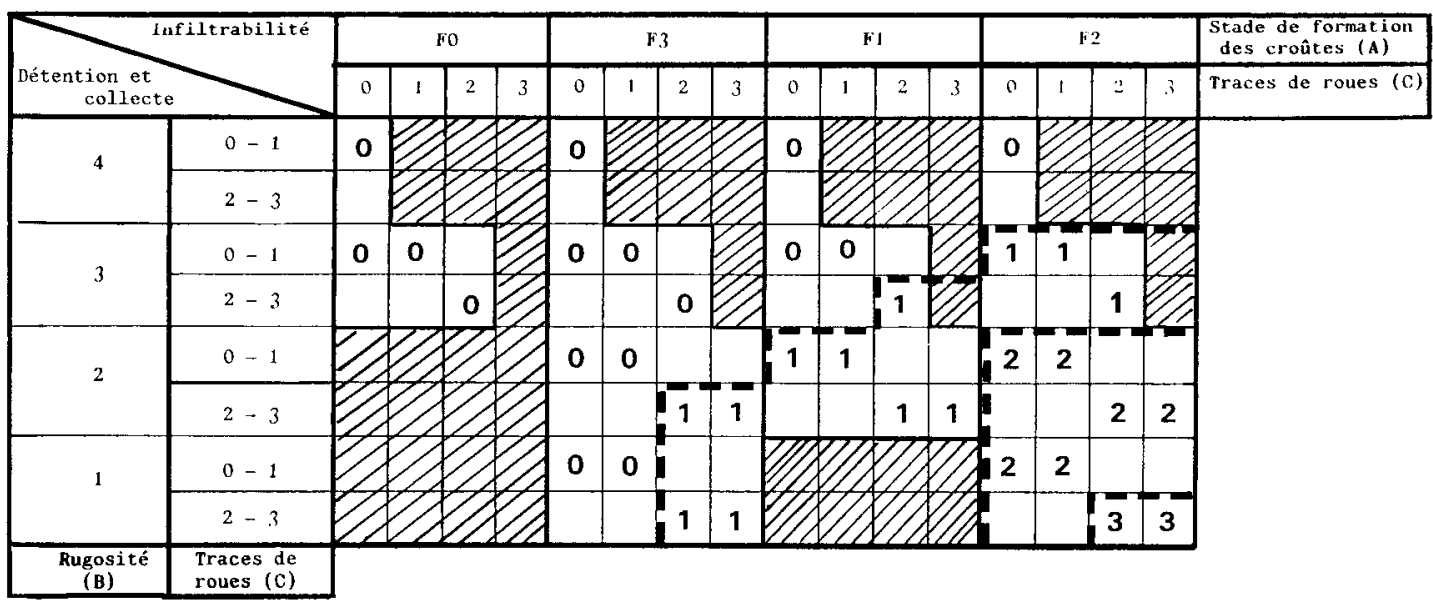

1) Les cases vides du tableau central correspondent à des impossibilités du fait que le critère atrace de roues » figure en ligne et colonne.

2) Comme le tableau est fait pour comparer des situations ou l'orientation des traces de roues par rapport au talweg, les dimensions des outils et tracteurs sont les mêmes, le rôle de collecte des roues n'est plus lié qu'à la note de traces de roues.

3) Les cases hachurces correspondent à des situations qui n’existent pas. 
de culture: ainsi sous blé, la dégradation structurale atteint le faciès 2 avant que la croissance du couvert ne soit suffisante pour jouer un rôle de protection. Aussi un champ de blé émet-il du ruissellement dès que l'intensité de pluie dépasse 1 à $2 \mathrm{~mm} / \mathrm{h}$ environ, même lorsque, une fois développée, la végétation recouvre le sol. Quant le sol est battu, le couvert n'empêche pas le ruissellement, tout au plus en réduit-il la vitesse. L'importance des

\section{TABLEAU 4}

Estimation de la date d'apparition du faciès $\mathrm{F}_{2}$ pour differentes dates de semis (BortFiN et al.. 1986).

Estimation of the date of appearance of the $F_{2}$ facies for different sowing dates.

\begin{tabular}{|c|c|c|c|c|}
\hline & Espèces & $\begin{array}{c}\text { Date } \\
\text { du semis }\end{array}$ & $\begin{array}{l}\Sigma P \text { pour } \\
\text { arriver } \\
\text { en phase } 2 \\
\text { (1) }\end{array}$ & $\begin{array}{c}\text { Date } \\
\text { estimec } \\
\text { d'appari- } \\
\text { tion de F2 }\end{array}$ \\
\hline \multirow{5}{*}{$\begin{array}{l}\text { Semis de } \\
\text { printemps }\end{array}$} & Pois & $10 / 03$ & $60 \mathrm{~mm}$ & $20 / 04$ \\
\hline & \multirow{2}{*}{ Lin } & $20 / 03$ & $60 \mathrm{~mm}$ & $30 / 04$ \\
\hline & & $10 / 04$ & $90 \mathrm{~mm}$ & $3 / 06$ \\
\hline & \multirow{2}{*}{$\begin{array}{l}\text { Betterave } \\
\text { Maïs }\end{array}$} & 20,04 & $90 \mathrm{~mm}$ & $10 / 06$ \\
\hline & & $30 / 04$ & $90 \mathrm{~mm}$ & 20,06 \\
\hline \multirow{4}{*}{$\begin{array}{c}\text { Semis } \\
\text { d'automne }\end{array}$} & \multirow[t]{2}{*}{ Escourgeon } & $30 / 09$ & $90 \mathrm{~mm}$ & $10 / 1:$ \\
\hline & & $10 / 10$ & $90 \mathrm{~mm}$ & $15 / 11$ \\
\hline & \multirow[t]{2}{*}{ Blé } & $10 / 11$ & $60 \mathrm{~mm}$ & $1 / 12$ \\
\hline & & $20 / 11$ & $60 \mathrm{~mm}$ & $10 / 12$ \\
\hline
\end{tabular}

(1) $60 \mathrm{~mm}$ pour les situations risquant peu de subir une dessiccation: $90 \mathrm{~mm}$ en cas contraire. traces de roues sous culture de blé étant faible à nulle (tabl. 2), la concentration du ruissellement est plus lente, toutes choses égales par ailleurs, que sous betterave ou après des chantiers de récolte de cultures de printemps (pomme de terre, betterave, maïs) qui laissent de nombreuses traces de roues. C'est bien la différence de délai nécessaire à la concentration qui est prise en compte dans l'attribution des notes 2 ou 3 .

\section{TABLEAU 5}

Estimation de l'évolution des classes de rugosité én fonction des pluies (umulees ( 2 Pmm) (BOIFFIN, 1984).

Evaluation of change in roughness grade with accumulated rainfall ( $\Sigma$ P mm).

\begin{tabular}{|c|c|c|c|c|}
\hline Classe & 4 & 3 & 2 & I \\
\hline $\begin{array}{l}\text { ¿Pmm néces- } \\
\text { saire pour faire } \\
\text { passer la rugo- } \\
\text { sité a la classe } \\
\text { inléricure }\end{array}$ & 100 & 150 & 200 & \\
\hline
\end{tabular}

Ce tableau n'est valable qu en l'absence de paille. L’atténuation de rugosité d'un déchaumage en cours d'hiver est très réduite.

CULTURES

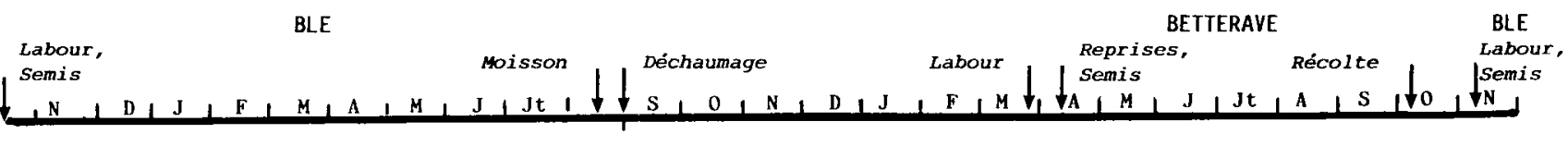

(A)

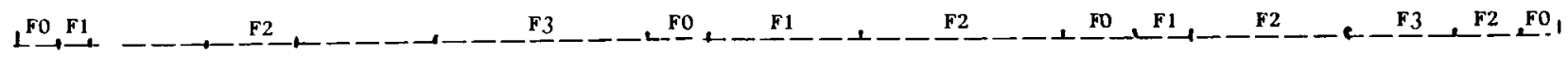

(B)

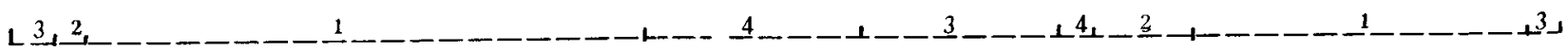

(c)

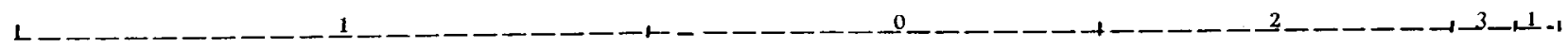

(D)

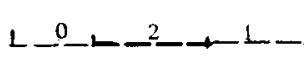

$\sum_{P m m}$

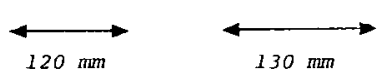

$\underline{0}$

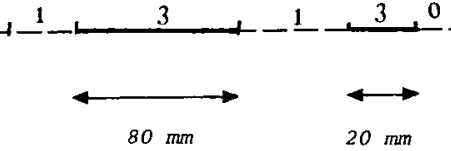

Figure 1

Evolution de l'aptitude d'unc parcelle à produire du ruissellement concentré (cas d'une succession ble-hetterave').

Note: Dans notre exemple, la betterave n'est pas binée mécaniquement, le déchanmage est mottear et cahotique.

Development of the ability of a plot to produce concentrated runoff (case of a winter wheat/sugar beet crop rotation).

Note: In this example sugar he't is not supposed mechanically we'eded, stubhle ploughing gives a rough surface.

Labour, Semis : Ploughing. Sowing.

Moissom: Hawest.

Déchanmage : Stubble plonghing.

Reprises, S'mis: Tillage', Sowing.

Récolte: Harvest.

Blè : Winter theat

Betterave: Sugar beet

Légende

Stade de développenent des contes de hartance : Crusting stage (A).

Classe de rugosité : Roughness grade (B).

Traces de roues: Wheel tracks (C).

Lex classes des differents critères soml domnées all lablean 2.

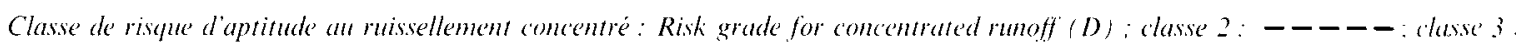


Reste maintenant à transformer l'estimation de l'aptitude à la concentration du ruissellement donnée par la procédure précédente en évaluation de risque de ruissellement concentré. Pour ce faire, on calcule le cumul des pluies sur les périodes où la note d'aptitude à la concentration est élevée (cf. $\Sigma$ P mm, fig. 1). Bien qu'on ne puisse admettre que pendant ces périodes toutes les pluies ruissellent, on peut faire l'hypothèse que cette dernière procédure classe les systèmes de culture selon les risques qu'ils entraînent sous un climat donné.

\section{RÉSULTATS ET DISCUSSION}

\section{A. Application à la comparaison de plusieurs successions culturales}

Aux différentes successions culturales données dans le tableau 1, nous avons appliqué la procédure de détermination des risques de ruissellement concentré telle qu'elle vient d'être explicitée. Pour les comparer entre elles, on adopte les conventions suivantes:

- On prend le régime pluviométrique moyen de Goderville établi par décade.

- On admet les dates de semis conventionnelles données au tableau 4, en choisissant, si besoin est, celle qui correspond le mieux à la date de libération de la parcelle, compte tenu du précédent cultural.

- On suppose que les betteraves ne sont pas binées, qu'il n'y a pas eu d'implantation d'engrais vert, que les seules cultures dérobées sont des espèces fourragères, et que ces agriculteurs ne réalisent pas de travaux du sol spécifiquement destinés à créer un état fragmentaire de surface dans le but d'augmenter l'infiltration, autant de modes de conduite dont on étudiera plus loin les effets.

Ces conventions étant les mêmes pour toutes les successions, leur comparaison est possible. Le tableau 6 donne pour chacune d'elles le cumul des pluies tombées pendant les périodes où les notes d'aptitude à la concentration sont 2 et 3 d'une part, 3 de l'autre. Les valeurs sont ramenées à l'année culturale par division de ces cumuls totaux de pluies sur chaque rotation par leur nombre d'années respectif.

Les différences de risques sont grandes entre les rotations: par rapport à la moyenne de pluviométrie annuelle, le cumul des pluies tombées pendant les périodes où les notes d'aptitude sont 2 ou 3 , varie de 11 à 45 p. 100 . Si l'on ne retient que les périodes à note 3 , la somme des pluies varie de 2 à 20 p. 100 .

Pendant la longue période hivernale (au demeurant la plus arrosée), comprise entre les semis de blé et la reprise des travaux de printemps, le pourcentage des différents états-types différencie nettement les successions. Dans les plus céréalières $(50$ p. 100$)$, où l'on peut alterner céréales d'hiver et cultures de printemps, il y a risque de ruissellement important un hiver sur deux (celui qui correspond à la culture du blé). Mais dans les successions moins céréalières, l'existence d'intercultures d'hiver entre deux cultures de printemps accroît le pourcentage des hivers où la surface émet du ruissellement. Aussi la variabilité des risques entre successions est-elle liée principalement à la proportion de ces intercultures (tabl. 6).
On rencontre les successions entraînant le plus de risques dans les exploitations où l'objectif d'augmentation de la production par unité de surface a fait adopter des cultures de pomme de terre et de légumes en plus de celles de la betterave et du lin. A l'inverse, les risques sont minimes dans l'exploitation qui, étant orientée vers l'intensification laitière sans cultures de vente, pratique des prairies de courte durée (18 mois) dans la succession, car ces dernières, semées tôt à l'automne, recouvrent vite le sol, empêchant ainsi la surface d'évoluer jusqu'au faciès $\mathrm{F} 2$

A noter cependant qu'il existe une autre différence (secondaire par rapport à la précédente) entre systèmes de culture : c'est la proportion de culture de printemps à semis précoce (pois et lin). Mis en place au moment d'un pic pluviométrique (fin mars-début avril), ces semis évoluent très vite vers un faciès F2 (tabl. 4).

Pour un assolement donné plusieurs successions de cultures sont envisageables, qui n'ont pas toutes forcément le même effet sur les risques de ruissellement. La rotation I (tabl. 1), qu'on rencontre dans les exploitations de type traditionnel, procède d'une logique de gestion de l'azote du sol. Le lin, craignant l'excès d'azote, est mis derrière deux céréales (blé et escourgeon), considérées à juste titre comme réduisant les reliquats azotés du sol, alors que l'apport de fumier est réalisé avant la betterave qui lui succède. Mais cette solution n'est pas la meilleure du point de vue des risques d'érosion, puisqu'elle entraîne l'existence d'une interculture lin-betterave, qui ruisselle en hiver, alors qu'en semant l'escourgeon derrière le blé on perd l'avantage d'une couverture du sol par les chaumes pendant une saison hivernale. Avec 50 p. 100 de céréales à paille, on pourrait réaliser une succession I' (BS-B1-L-Esc$\mathrm{BS}-\mathrm{Bl}$ ), dont les risques de ruissellement seraient pratiquement identiques à ceux de la succession II-a.

\section{B. Application à la comparaison de plusieurs techniques culturales}

Pour comparer des successions culturales nous avons été amenés à supposer des modes de conduite identiques de chaque culture. Or dans la pratique, il en existe plusieurs et on peut en proposer de nouveaux qui ne remettent pas en cause les choix d'assolement. Nous allons appliquer la même démarche pour classer plusieurs itinéraires techniques. Nous prendrons 2 situations :

1) Les intercultures d'hiver, dont nous avons souligné l'importance dans la variabilité des risques.

2) La culture de betterave.

Le jugement ne doit pas se limiter aux risques de ruissellement concentré, mais apprécier les états du sol dans le collecteur principal qui, s'ils sont meubles, accroissent les risques de départ de terre.

\section{Comparaison de différentes conduites d'intercultures}

La figure 2, établie selon les mêmes principes que la figure 1, permet de comparer différentes intercultures d'hiver, d'après l'évolution d'état de la surface du sol.

a) Derrière paille les deux modes de conduite (déchaumage et non-intervention), pour des raisons 
TABLEAU 6

Comparaison des risques de ruissellement concentré pour différents types de successions culturales.

Comparison of the risk of concentrated runoff for different crop rotaitons.

\begin{tabular}{|c|c|c|c|}
\hline \multirow[t]{2}{*}{$\begin{array}{l}\text { Numéro de } \\
\text { référence } \\
\text { des } \\
\text { successions } \\
\text { culturales } \\
\text { (tabl. } 1 \text { ) }\end{array}$} & \multicolumn{2}{|c|}{$\begin{array}{l}\sum P(\mathrm{~mm}) \text { tombée } \\
\text { en moyenne pendant un an } \\
\text { au cours des périodes } \\
\text { où l'aptitude } \\
\text { au ruissellement } \\
\text { prend les notes: }(1)\end{array}$} & \multirow[t]{2}{*}{$\begin{array}{l}\text { Importance } \\
\text { relative } \\
\text { des } \\
\text { intercultures } \\
\text { d'hiver } \\
\text { après } \\
\text { récolte } \\
\text { d'automne }\end{array}$} \\
\hline & 2 et 3 & 3 seulement & \\
\hline I & $\begin{array}{c}255 \\
30 \%\end{array}$ & $\begin{array}{l}35 \\
4 \%\end{array}$ & $1 / 6$ ans \\
\hline II A & $\begin{array}{c}170 \\
20 \%\end{array}$ & $\begin{array}{l}20 \\
2 \%\end{array}$ & $0 / 6$ ans \\
\hline II B & $\begin{array}{c}300 \\
36 \%\end{array}$ & $\begin{array}{l}75 \\
9 \%\end{array}$ & $2 / 6$ ans \\
\hline III A & $\begin{array}{c}315 \\
37 \%\end{array}$ & $\begin{array}{l}110 \\
13 \%\end{array}$ & $2 / 6$ ans \\
\hline III B & $\begin{array}{c}380 \\
45 \%\end{array}$ & $\begin{array}{l}175 \\
20 \%\end{array}$ & $4 / 8$ ans \\
\hline IV A & $\begin{array}{c}245 \\
29 \%\end{array}$ & $\begin{array}{l}165 \\
19 \%\end{array}$ & $1,5 / 4$ ans \\
\hline IV B & $\begin{array}{c}245 \\
29 \%\end{array}$ & $\begin{array}{l}130 \\
16 \%\end{array}$ & $2 / 6$ ans \\
\hline V & $\begin{array}{c}95 \\
11 \%\end{array}$ & $\begin{array}{l}35 \\
4 \%\end{array}$ & $0 / 5$ ans \\
\hline
\end{tabular}

(1) Les calculs ont été faits pour une pluviométric moyenne de $850 \mathrm{~mm}$. Le pourcentage par rapport à cette valeur est donné en italique.

différentes, n'engendrent pas de risques. Dans le premier cas, le maintien tout au long de l'hiver d'une rugosité suffisante fait obstacle à la mise en mouvement de l'eau ; dans le second, c'est la bonne infiltrabilité du sol. Le faciès F3 correspondant à cette situation est caractérisé par l'existence d'une fissuration due vraisemblablement à des phénomènes physiques (humectationdessiccation; gel-dégel), mais aussi biologiques. Aussi n'observe-t-on que très rarement du flaquage en surface sur chaumes non retournés.

Si le non-déchaumage est si peu pratiqué, c'est qu'il ne permet pas une aussi bonne décomposition ultérieure de la paille qu'un pré-mélange par déchaumage. De plus, le contrôle des adventices doit se faire exclusivement par voie chimique, ce qui est généralement coûteux. Toutefois, dans la zone de collecte principale (talweg), le déchaumage est à déconseiller car une couche de surface travaillée est meuble, donc peu résistante à la friction exercée par l'écoulement. Une solution consistant à déchaumer la parcelle, excepté l'axe du talweg sur une largeur de quelques mètres, constitue un bon compromis, relativement aisé à réaliser avec un outil porté.

b) Après récolte de pomme de terre et de betterave les risques de ruissellement sont très grands pendant toute la période hivernale si le sol n'est pas travaillé. Il en est de même après la récolte du lin, bien que les traces de roues aient un moindre rôle de collecte.

En travaillant le sol, on lui confère une infiltrabilité et une capacité de détention de l'eau en surface plus élevée. L'infiltrabilité diminue vite car, à cette époque très pluvieuse de l'année, l'évolution des croûtes de surface est rapide. C'est donc essentiellement grâce au maintien d'une rugosité suffisante que cette pratique peut avoir un rôle anti-érosif. Sur la figure 2 , nous avons représenté l'évolution des états de surface correspondant à deux dates de travail du sol $(1 / 11$ et $1 / 12)$, en utilisant les références des tableaux 4 et 5 . Calculé sur la base des pluviométries moyennes, le cumul des pluies pendant les périodes à risques élevés (classes 2 et 3 ), ne permet pas de différencier l'effet des 2 dates de travail. A la plus précoce correspond une dégradation de la rugosité qui, à partir du mois de février, ne permet plus une détention suffisante pour éviter le ruissellement. Mais si l'on travaille tard, le risque est élevé tant que l'opération n'est pas réalisée. Une analyse fine du comportement des différentes situations d'interculture hivernale a été faite récemment par OUVRY (1987).

Dans la pratique, le travail après récolte d'automne est rare. En l'absence de résidus importants il n'a d'autre fonction qu'anti-érosive. Comme il constitue une des voies de progrès pour limiter les risques d'érosion, il nous parait devoir être envisagé. Une fois les semis de blé achevés, les agriculteurs disposent d'une période pour exécuter une telle opération, particulièrement dans les exploitations possédant le plus de surfaces justiciables de ce traitement (Type III), où les semis de blé sont finis le plus tôt puisque la sole emblavée y est plus réduite. A noter cependant que cette période est utilisée pour réaliser des épandages d'amendement calcique ou de fumier. Ceci est particulièrement vrai entre la récolte de lin et le semis de betterave. Or travailler le sol diminue la portance. Aussi doit-on préconiser de faire les épandages le plus tôt possible, dans la mesure où les stocks de fumier sont disponibles, avant de faire un travail motteux et chaotique (OUvry, 1987).

c) Les espèces en culture dérobée (engrais verts ou cultures fourragères) peuvent avoir un rôle anti-érosif si elles sont semées assez tôt (ou si elles ont une vitesse de croissance suffisante au départ) pour arriver à recouvrir le sol avant que l'évolution des croûtes n'ait atteint le faciès F2. La figure 2 illustre la différence de risque selon que la culture a recouvert ou non le sol à temps.

La pratique des engrais verts ne peut done s'envisager que derrière un précédent libérant tôt le sol. C'est pour cela qu'on les rencontre souvent derrière le blé. Si l'on compare le rôle des engrais verts à celui des chaumes, retournés ou non, on constate qu'ils ne jouent pas au niveau de l'impluvium un rôle anti-érosif déterminant. Aussi est-ce plutôt après les cultures de printemps qu'on doit chercher à justifier leur emploi.

Derrière une culture de pois le problème se pose peu, cette culture étant presque toujours suivie de blé. On peut envisager d'installer utilement des engrais verts après l'enlèvement $\mathrm{du}$ lin ou les premières récoltes de pomme de terre du mois de septembre. On doit alors choisir des espèces à croissance rapide et des modes d'installation peu coûteux en temps (OUvRY, 1987). En revanche, après récolte tardive de pomme de terre, après betterave et mais, il n'est pas question de compter sur 


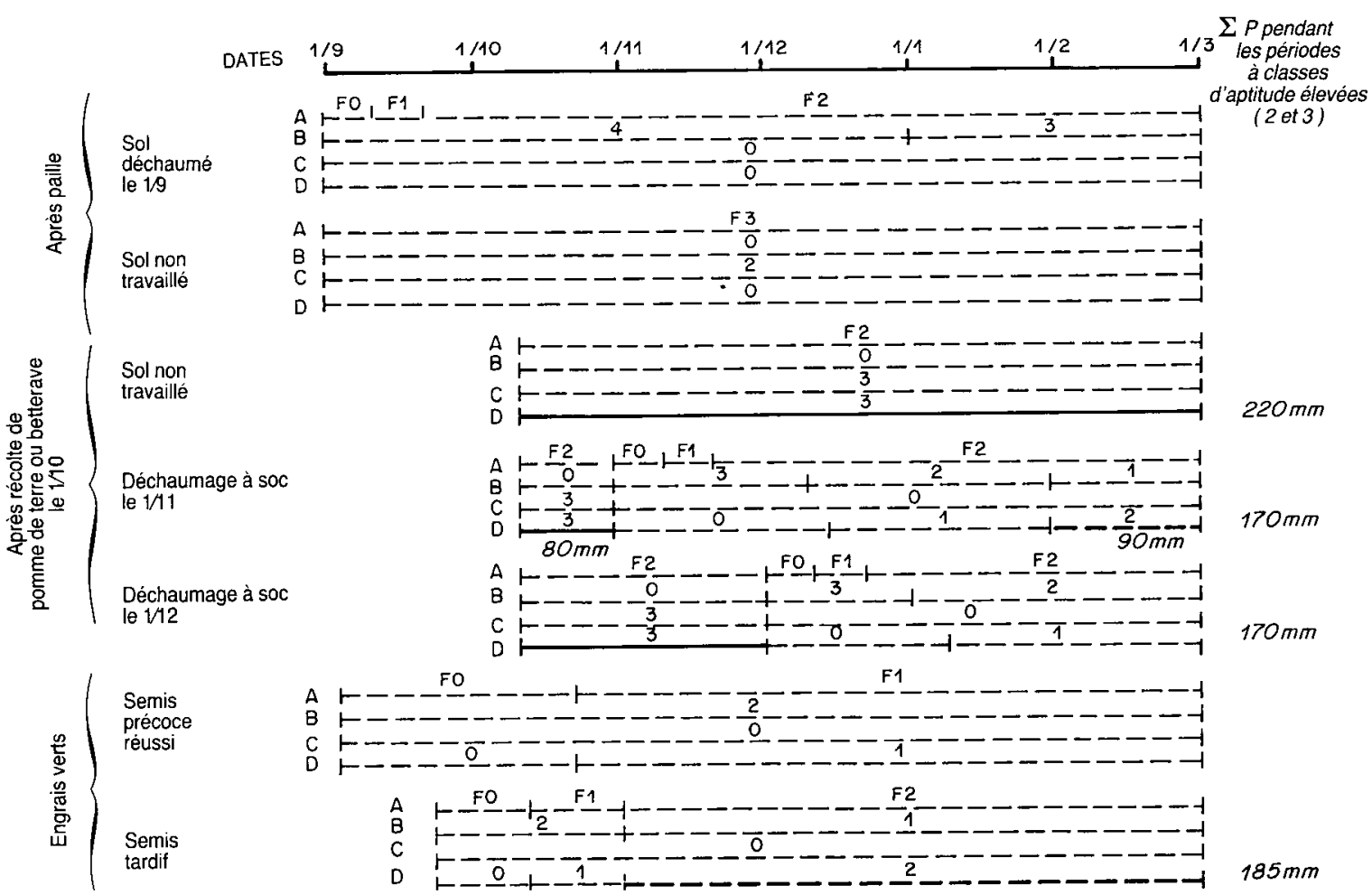

Figure 2

Evolution de l'aptitude d'une parcelle à générer du ruissellement concentré (D) selon différents modes de conduite des intercultures hivernales. Development of the ability of a plot to produce concentrated runoff for different soil managements in winter.

Légende : $A, B, C, D:$ cf. figure 1 .

Les classes des diffërents critères sont données au tableau 2.

cette technique pour limiter le ruissellement. C'est le travail du sol qui est à préconiser.

Dans la pratique, l'introduction des engrais verts dans les exploitations à pomme de terre et légumes (Type III) se heurte à un obstacle : l'emploi du temps très chargé pendant le mois de septembre.

\section{Comparaison de différentes conduites de la betterave}

Les parcelles cultivées en betterave se caractérisent par une faible rugosité et des traces de roues relativement nombreuses (note 2, tabl. 2). La surface de ces dernières, dont l'infiltrabilité est plus faible que celle des inter-roues, peut être suffisante pour déclencher du ruissellement ; toutefois c'est généralement à partir de l'apparition du faciès $\mathrm{F} 2$ dans les zones inter-roues qu'augmentent les risques d'érosion. Aussi la date de semis a-t-elle un effet notable sur le phénomène.

La figure 3 illustre l'effet d'une différence de date. Elle a été établie à partir de références sur la croissance de la betterave (FLEURY, communication orale) et sur la vitesse d'évolution des croûtes, différenciées selon que le sol est soumis ou non à une période de dessiccation (BoIfFIN et al., 1986). En l'absence de binage, pour un semis du 20/03, le faciès F2 apparaît dès le $10 / 04$, bien avant le début de croissance rapide de la betterave (le 5/06). Pour un semis du 20/04, le ruissellement se déclenche le 20/06, quelques jours à peine avant l'accroissement du couvert. Dans un cas comme dans l'autre, le processus de dégradation ne peut être interrompu par un effet de protection du feuillage, mais l'effet de la date de semis se manifeste par une forte différence de cumul des pluies tombées entre l'apparition du faciès $\mathrm{F} 2$ et la fin juillet, date à laquelle on suppose que le remaniement des croûtes par fissuration (faciès F3) et la dessiccation du profil peuvent améliorer sensiblement l'infiltration.

Les dates de semis sont retardées par une forte pluviométrie de mars, mais plus nettement là où les soles de pois et de lin, semées avant la betterave, sont importantes (Type II). En revanche dans le Type III, où l'agriculteur a des pommes de terre à planter, la tendance est à avancer les semis de betterave.

Dans les itinéraires techniques observés, au contraire de ce que nous avons supposé jusqu'à présent, le binage est courant (26 exploitations sur 30 ). Associé à un seul traitement chimique il permet un contrôle économique des adventices. Réalisé avec des outils à dents verticales (cf. plus loin), il augmente l'infiltrabilité et joue un rôle anti-érosif quand il est pratiqué dans l'impluvium. Dans la zone de concentration, par contre, son effet est néfaste. Comme dans l'exemple précédent du déchaumage les deux zones sont justiciables de traitements différents. Sur la base des données de la figure 3, on peut estimer qu'un seul binage suffit à arrêter le processus de ruissellement en cas de semis tardif en obtenant un effet protecteur du couvert avant le retour de la surface du sol au faciès $\mathrm{F} 2$; deux interventions seraient sans doute nécessaires pour obtenir le même effet en semis précoce.

Il convient cependant de préciser que les bineuses classiques à rasettes sont dangereuses car elles créent un lissage à $3-4 \mathrm{~cm}$ de profondeur. On préfèrera des outils à dents verticales. 
Mais dans certaines exploitations la pratique du binage est impossible pour des questions d'organisation du travail. Sur les quatre exploitations qui n'en font pas, trois sont des exploitations de grande culture intensive (Type III), où les buttages de pommes de terre, l'entretien de la sole de légume viennent concurrencer cette opération. Elle y serait pourtant d'autant plus utile que le semis de betterave est précoce. La quatrième exploitation (Type II) a le ratio surface de betterave/poste de travail le plus élevé de notre échantillon (16 ha), valeur supérieure au seuil établi par d'autres auteurs dans des systèmes de production similaires (CAPILLON et al., 1986) à partir duquel l'agriculteur n'a pas le temps de réaliser des binages et doit compter uniquement sur l'effet des désherbages chimiques faits en post-levée.

\section{L'affectation des cultures au parcellaire d'un bassin versant}

Nous avons jusqu'à présent considéré des bassins versants simples, constitués d'une seule parcelle culturale. Mais les parcelles n'étant généralement pas isolées hydrauliquement dans les paysages d'open field, les risques d'érosion les plus importants apparaissent quand une parcelle, dont l'état du sol est compact, domine une autre qui vient d'être ameublie. C'est le cas des parcelles de betteraves ou de pommes de terre après récolte (note de risque 3) dominant des semis de blé à l'automne ou de parcelles de blé (note de risque 2) dominant des semis de pois, lin, ou betterave au printemps. A l'inverse, les risques sont nuls à faibles tant que les positions hautes son occupées par des parcelles fraîchement travaillées.

\section{Présentation d'un cas}

Soit le cas présenté sur la figure 4 , situation réellement observée d'un bassin versant isolé hydrauliquement, cultivé par un seul agriculteur. Il correspond à une rotation culturale classique d'un blé suivi de deux cultures de printemps. L'agriculteur a fait se succéder les différentes cultures comme indiqué au tableau $\mathrm{A}$ de la figure 4. Dans un souci de commodité, il a regroupé les deux parcelles de blé et a donc aménagé le bassin versant en trois blocs.
La figure 5-A présente de façon schématique, sur trois années consécutives, les états de surface des différentes parcelles, au printemps et en automne, juste après que les semis ont été réalisés. Elle permet de constater que deux fois sur trois, pour chacune des deux saisons, un impluvium constitué de deux parcelles susceptibles de ruisseler domine des parcelles fraîchement ameublies. II s'agit là de configurations à risques d'érosion très élevées.

\section{Discussion}

Deux propositions nous paraissent tout à fait compatibles avec la conduite des systèmes de culture. Elles consistent :

a) à éviter de regrouper en un bloc de culture des parcelles voisines, comme tel est souvent le cas pour le blé dans les successions pratiquées (il faudrait cependant estimer la taille au-dessus de laquelle le gain de productivité du travail est faible au regard des inconvénients sur l'érosion);

b) à réduire la fréquence des situations à risques. S'il est impossible d'y parvenir au printemps, où l'on ne peut éviter d'avoir des semis dominés par du blé, c'est tout à fait possible en automne. Les parcelles semées en blé peuvent être systématiquement situées en aval de chaumes plutôt que de parcelles venant de subir des récoltes, à condition qu'au cours des années successives l'affectation des parcelles au blé se fasse de haut en bas du bassin versant.

La proposition d'affectation des cultures aux parcelles, que nous faisons dans le tableau B de la figure 4, combine les 2 propositions précédentes. Sur la figure 5-B correspondant à ces nouvelles règles d'affectation, nous pouvons faire les constatations suivantes.

1) Au printemps, le nombre de situations à risque augmente, mais pour chacune d'elles la surface de l'impluvium est 2 fois plus petite. Il s'ensuit que la quantité susceptible de ruisseler et de se concentrer dans le talweg en aval de la parcelle est deux fois plus faible, et, en régime continu, la vitesse d'écoulement également. Or, le détachement des particules en fond de talweg n'est obtenue qu'au-dessus d'une vitesse critique (Govers, 1985 ; Govers \& Rauws, 1986). En dimi-
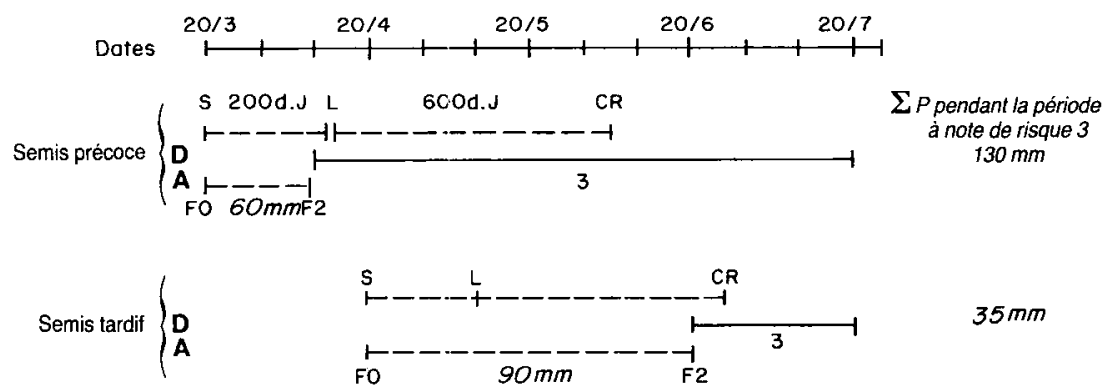

Figure 3

Comparaison des aptitudes a la concentration du ruissellement pour deux dates de semis de betterave.

Comparison of concentrated runoff ability for two sugar beet sowing dates.

Note : $d . j$ : Degrés . Jour

$S:$ Semis.

L: Levée.

$C R$ : Début de croissance rapide.

Les autres symholes sont donnés au tabléau 2 et à la figure 1. 
nuant de moitié la taille des parcelles, on réduit sensiblement les risques d'érosion en aval.

2) Il n'existe pas de situations à risques en automne. Certes on rencontre des cas où des parcelles en interculture après récolte d'automne dominent des chaumes. Mais, plus anciennement travaillés que les parcelles en blé, ceux-ci résistent mieux à l'incision par le ruissellement concentré; plus rugueux, ils retiennent mieux l'eau.

En conséquence, à condition d'accepter de ne pas agrandir exagérément les parcelles et de prévoir les situations de superposition entraînant des risques, il est possible de limiter ces derniers par quelques règles simples d'affectation des cultures aux parcelles.

\section{CONCLUSION}

L'importance majeure des états de l'impluvium dans le processus d'érosion étudié nous a incités à proposer une procédure d'estimation des risques de ruissellement concentré à partir de l'évolution de ces états. Qualitative, la procédure utilisée ne peut servir qu'à comparer les effets de systèmes de culture qui seraient appliqués à une même situation géomorphologique avec des formes et tailles de parcelles identiques, une même orientation du réseau de collecte et un même climat. Empirique, elle a un domaine de validité qui est celui des références à

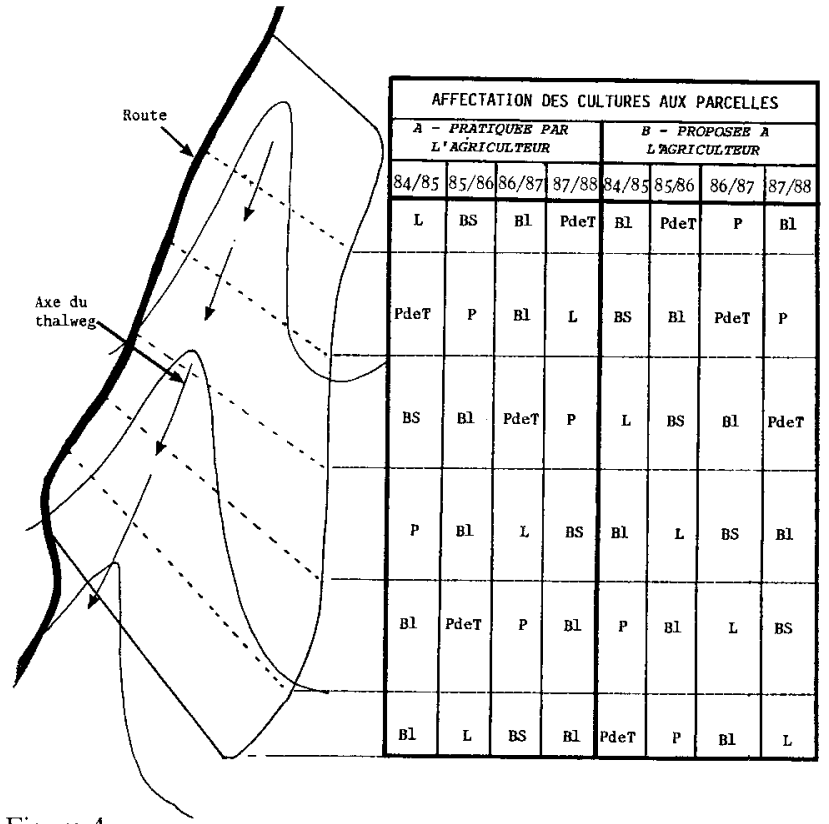

Figure 4

Deux modes d'affectation des cultures au parcellaire d'un bassin versant, pour une rotation betterave-blé-pomme de terre-pois-blé-lin.

Note :

Les symboles correspondent à ceux employés au tableau 1 .

La surface de chaque parcelle élémentaire est de 6 ha environ ; la pente du talweg inférieure à 4 à 5 p. 100

Two manners of allocating field crops to the plots of a catchment area for the crop rotation : sugar beet-winter wheat-potato-pea-winter wheatflax.
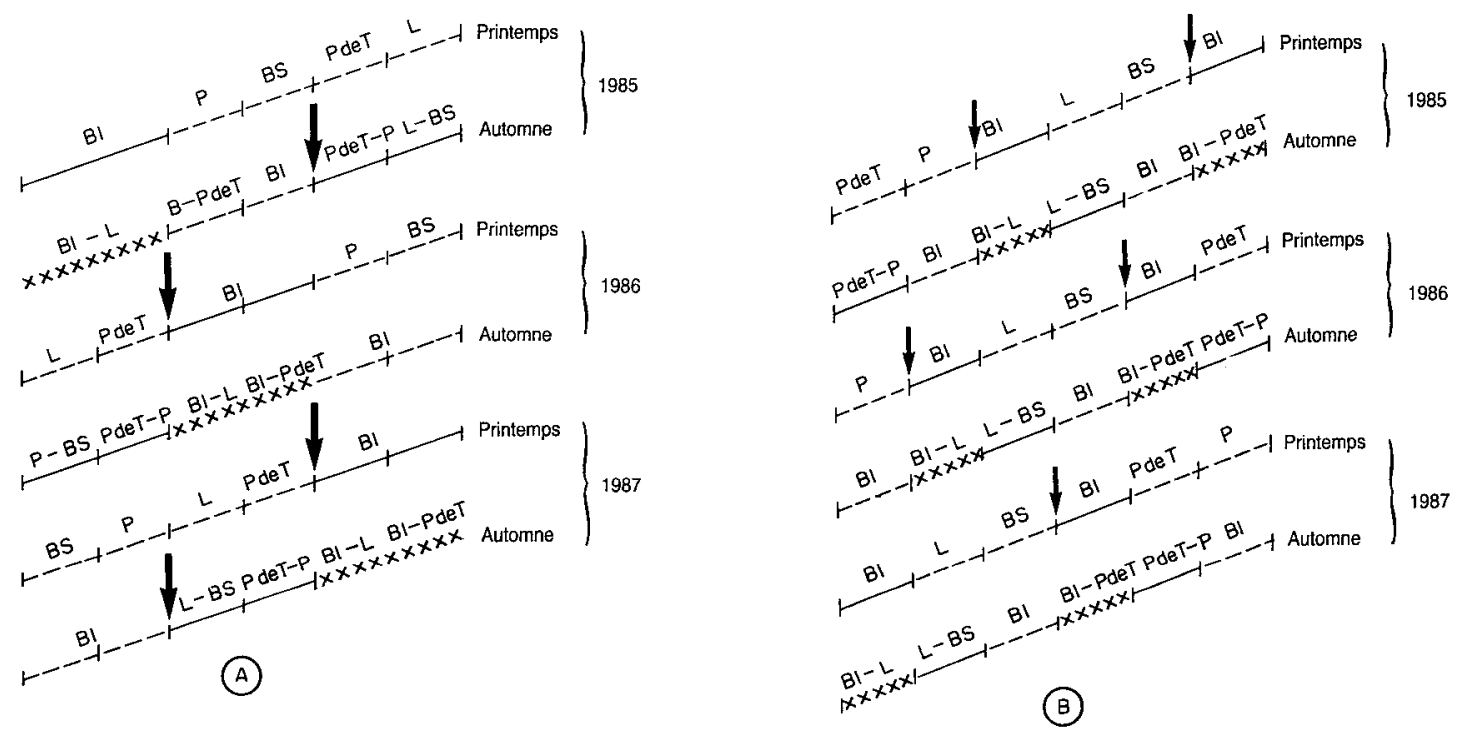

Figure 5

Evolution des états de surface des parcelles du bassin versant représenté sur la figure 4, selon 2 modalités d'affectation des cultures aux parcelles. A : pratiquée par l'agriculteur.

B : proposée.

Change in the surface state fields in the catchment area shown in figure 4, according to 2 modes of allocating crop production.

A : practised by farmer.

$B:$ proposed.

Note: Les symboles correspondent à ceux employés au tableca 1 .

Légende: $\quad$ Surface émettant du ruissellement.

Surface area producing runoff.

Surface fraîchement travaillée.

Surface area just tilled.

Chaume Straw.

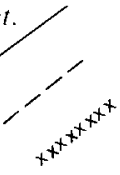

Risque d'érosion important (grand impluvium).

Major erosion risk (big catchment area).

Risque d'érosion faible (petit impluvium).

Low erosion risk (small catchment area). 
partir desquelles elle a été établie: limon battant sur faible pente, pluies de faible intensité. Elle nous a cependant permis de répondre aux questions posées:

- Les types d'assolements recensés présentent des différences appréciables de risques liées, pour une grande part, à l'importance relative des surfaces susceptibles de ruisseler pendant la période hivernale.

Un certain nombre de techniques, compatibles avec les assolements choisis, sont susceptibles de réduire le ruissellement, tout particulièrement pendant les périodes d'intercultures.

Retenons toutefois que l'importance majeure des états de l'impluvium dans ce processus érosif conduit à des conclusions sensiblement différentes des règles couramment admises. C'est ainsi, par exemple, que les engrais verts ne sont efficaces que sous certaines conditions de semis précoces et leur intérêt n'est pas décisif si on les compare à du déchaumage. Des techniques d'ameublissement du sol (comme le binage des betteraves, le travail des intercultures), souvent réputées dangereuses, peuvent au contraire limiter les risques moyennant quelques précautions dans les zones de concentration.

Deux points ressortent de cette étude, importants à souligner pour la maîtrise des risques d'érosion.

1) Il n'est pas possible, au moyen des techniques culturales, de réduire entièrement le ruissellement: les parcelles cultivées en blé, colza, lin, pois, par exemple, une fois les croûtes de battances bien développées, ruissellent sans qu'on puisse, par une opération de fragmentation du sol, arrêter le phénomène. Le ruissellement est, pour partie, inéluctable. Aussi la stratégie de lutte contre l'érosion dans les situations comparables à celles du Pays de Caux doit combiner la gestion des états de surface des parcelles, des règles de dimensionnement de ces dernières et d'affectation des cultures dans le parcellaire à des aménagements hydrauliques du bassin versant. Dans leurs principes, ces derniers consistent :

- à isoler hydrauliquement les différentes parcelles constituant un impluvium,

- à canaliser le ruissellement dans les zones de concentration aménagées pour limiter son effet érosif,

- à intercepter le ruissellement dans des bassins de retenue en amont des secteurs d'habitat à protéger.

2) Pour des raisons d'organisation du travail propres aux différents types d'exploitations rencontrés, il n'est pas possible de préconiser les mêmes techniques à chacun d'eux.

Aussi un nouveau champ de recherche s'ouvre-t-il, visant l'introduction de techniques de maittrise du ruissellement et de l'érosion dans le fonctionnement des exploitations ainsi que dans la gestion collective des bassins versants.

Recule 10 mars 1988 Accepté le 8 juillet 1988

\section{REMERCIEMENTS}

Le financement de cette étude a été assuré par le Ministère de l'Agriculture (DIAME), et le Service d'Economie Régionale et le Développement Agricole de Haute-Normandie.

Nous tenons à remercier Anne-Véronique AuzeT, Jean-François Ouvry el Pierre StengFl pour les précieuses remarques qu'ils nous ont faites sur le premier manuscrit.

\section{RÉFÉRENCES BIBLIOGRAPHIQUES}

Auzet A. V., 1987. L'érosion des sols par l'eau dans les régions de grande culture: aspects agronomiques. Min. Env./Min. Agric., CEREG, Organisation-Environnement. 53 p. + Annexes.

Boiffin J., 1984. La dégradation structurale des couches superficielles du sol sous laction des pluies. Thèse Docteur Ingénieur. INA-PG, 320 p. + Annexes.

Boiffin J., Monnier G., 1986. Infiltration rate as affected by soil surface crusting caused by rainfall, 210-217. In F. Callebaut, D. Gabriels, M. de Boodt. Assessment of soil surface sealing and crusting. Flanders Research Center for Soil Erosion and Soil Conservation, Ghent (Belgium), $374 \mathrm{p}$.

Boiffin J., Papy F., Peyre Y., 1986 : Sistèmes de production, rystemes de culture ét risques d'erosion dams le Pays de Caux. Rap. dact. INRA/INA-PG/Min. Agric./DIAME, 154 p. + Annexes.

Boiffin J., Papy F., Eimberck M., 1988. Influence des systèmes de culture sur les risques d'érosion par ruissellement concentré. I Analyse des conditions de déclenchement de l'érosion. Agrononie, 8 (8), 663-673

Bolline A., 1977. La vitesse de l'érosion sous culture en région limoneuse. Pidologic, 27 (2), 191-206.

Capillon A., 1986. A classification of farming systems, preliminary to an extension program. A methodology; Proc. $5 \mathrm{th}$ Faming Systems Symp, Kansas State Univ., 219-235.

Capillon A., Fleury A., Leterme Marie-José, 1986. Déterminants des pratiques agricoles et conception d'un dispositif expérimental. Bull. Tech. Inf., Min. Agric., 408, 271-279.

Capillon A., Manichon H., 1988. Guide d'étude de l'exploitation à liusage de lagronome. Relance Agronomique, ADEPRINA/APCA, $61 \mathrm{p}$.

De Ploey J., 1986. Soil erosion and possible conservation measures in loess loamy areas, 157-163. In G. Chisci, R. P. C. Morgan. Soil erosion in Earopean Community, impact of changing Agriculture. Balkema, Rotterdam, Boston, $233 \mathrm{p}$.
Foster G. R., Lane L. J., Mildner W. F., 1985. Seasonally ephemeral cropland gully crosion. Proc. of the ARS-SCS Natural resources modelling workshop. Washington, D.C./USDA-Agricultural Research Service.

Fullen M. A., 1985. Compaction hydrological processes and soi crosion on loamy sands in east Shropshire, England. Soil Tillage Res., $6,17-29$.

Govers G., 1985. Selectivity and transport capacity of thin flows in relation to rill erosion. Catena, 12, 35-49

Govers G., Rauws G., 1986. Transporting capacity of overland flow on plane and irregular beds. Earth surface processes, 11, 515-524.

Monnier G., Boiffin J., Papy F., 1986. Réflexions sur l'érosion hydrique en conditions climatiques et topographiques modérées : cas des systèmes de grande culture de l'Europe de l'Ouest. Cah. ORSTOM, Sér. Pédol., XXII (2), 123-131.

Ourry J. F., 1987. Bilan des travaux, Campagne 1986-1987. AREAS, rap. 153 p. + Annexes.

Pinguet A., 1985. Aperçu sur la pluviométric en Pays de Caux. Doc. int. Bioclimatologie, INRA, Avignon, $5 \mathrm{p}$.

Rauws G., Auzet A. V., 1988. Laboratory experiments on the effects of simulated tractor wheelings on linear soil erosion (à paraitre dans Soil (and Tillage Res.).

Savat J., De Ploey J., 1982. Sheet wash and rill development by surface flow, 113-126. In R. Bryan, A. Yair, Badland Geomorphology and Piping, Geobooks, Cambridge, $218 \mathrm{p}$.

Spomer R. G., Hjelmfelt A. T., 1986. Concentrated flow erosion on conventional and conservation tilled watersheds. Trans $A S A E, 29$ (1), 124-127. 